Supporting Information

\title{
Adsorption/Desorption of Mono- and Diblock Copolymers on Surfaces using Specific Hydrogen Bonding Interactions
}

Amitav Sanyal, Tyler B. Norsten, Oktay Uzun, and Vincent M. Rotello*

Department of Chemistry, University of Massachusetts, Amherst, MA 01003, USA.

1.1 XPS analysis of SAM 1 : before and after deposition of polymers.

SAM1
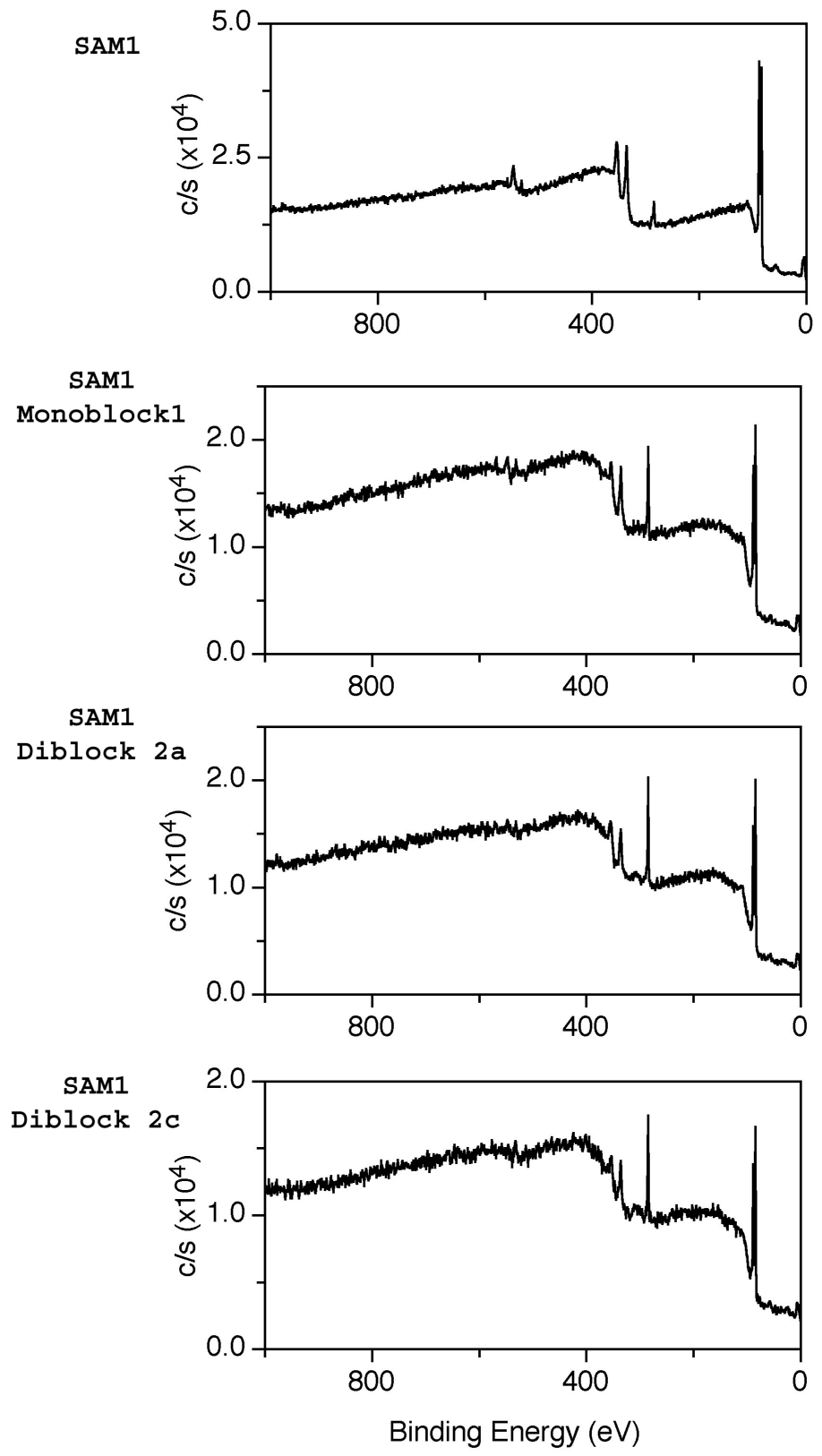

For expanded plots of multiplex scans of $\mathrm{Au}$ and $\mathrm{C}$ regions see Figure 3a (main text). 
1.2 XPS analysis of SAM 1 : before and after heating in 1:3 $\mathrm{EtOH} / \mathrm{CHCl}_{3}$ for $10 \mathrm{~min}$. (Ref 21)
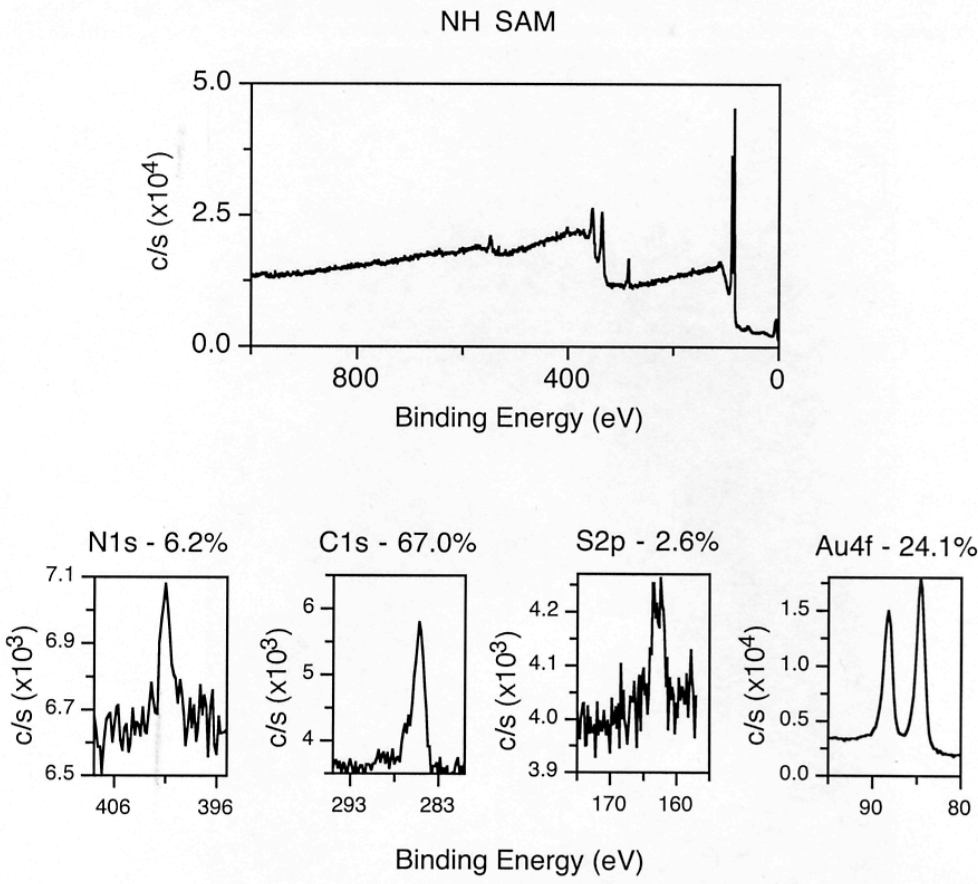

After 10 min desorption 60 deg $1: 3 \mathrm{EtOH} / \mathrm{CHCl}_{3}$
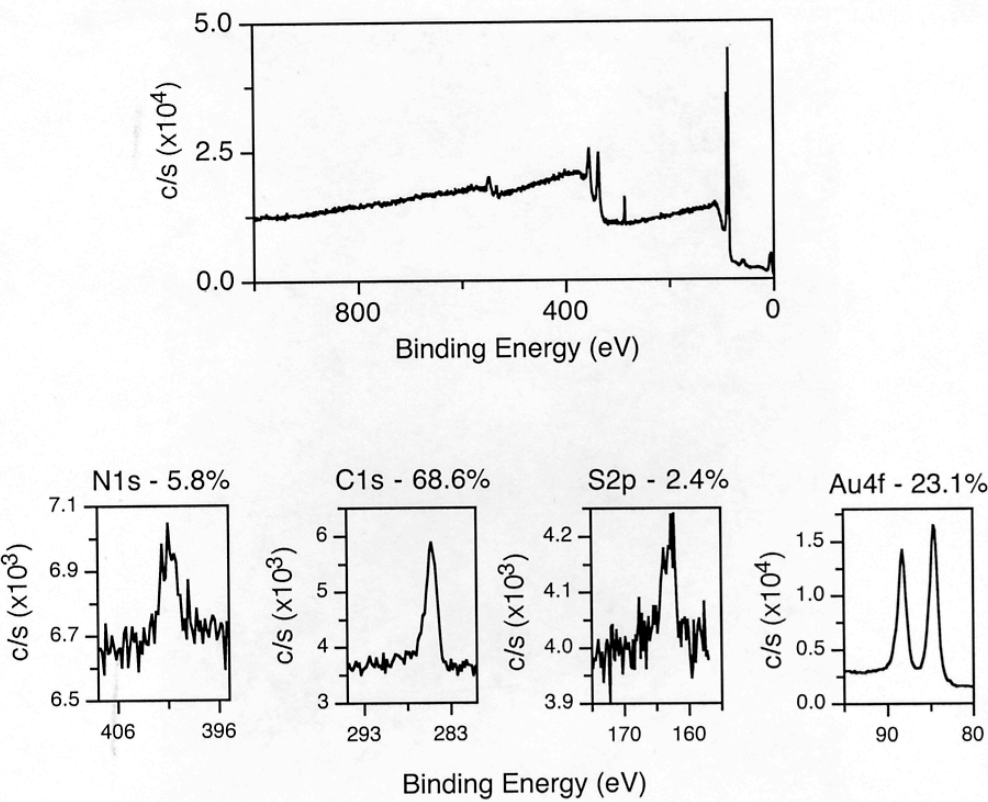

Figure above shows that monolayer composition of SAM1 remains similar before and after desorption protocol. 
1.3 Thickness and contact angle measurement at every step of adsorption/ desorption/re-adsorption of monoblock copolymer 3 on SAM1 and control SAM2.

(Ref 20)

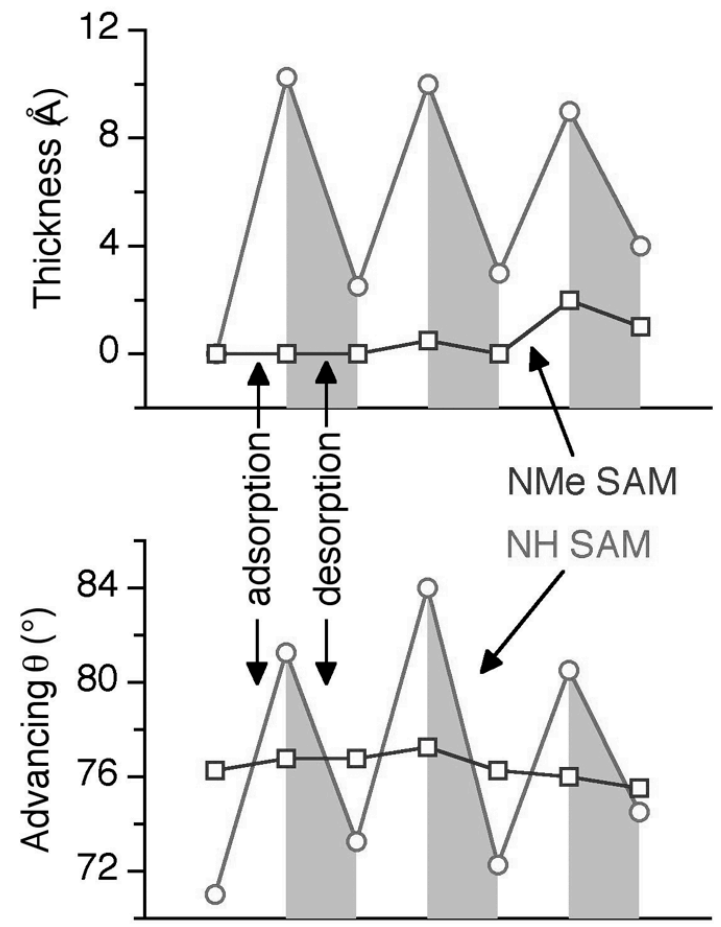

Figure above shows changes in the thickness of polymer film obtained using monoblock copolymer 3 on SAM1 and advancing contact angle at each stage of surface modification study. It also demonstrates that subjecting the control surface SAM2 to similar deposition/desorption processes does not result in any modifications.

1.4 Number molecular weights $\left(M_{n}\right)$, polydispersity index (PDI) and the \% of chloromethylstyrene (i.e functionalization) in the 'adhesive block' of the various polymers utilized.

\begin{tabular}{c|c|c|c|c} 
Polymer & $\begin{array}{c}\mathrm{M}_{\mathrm{n}} \text { block A } \\
(\mathrm{kg} / \mathrm{mol})\end{array}$ & $\begin{array}{c}\mathrm{M}_{\mathrm{n}} \text { block B } \\
(\mathrm{kg} / \mathrm{mol})\end{array}$ & PDI $^{\mathrm{b}}$ & $\begin{array}{c}\text { \% of CMS in } \\
2^{\text {nd }} \text { block }\end{array}$ \\
\hline $\mathbf{1}$ & 0 & 12 & 1.20 & 18 \\
$\mathbf{2 a}$ & 27 & 7 & 1.14 & 20 \\
$\mathbf{2 b}$ & 27 & 13 & 1.26 & 17 \\
$\mathbf{2 c}$ & 27 & 27 & 1.26 & 17 \\
$\mathbf{2 d}$ & 13 & 13 & 1.16 & 16 \\
$\mathbf{4}$ & 29 & 11 & 1.22 & 17
\end{tabular}

${ }^{a}$ All polystyrene monoblock polymers used to synthesise various diblock copolymers had PDI ca. 1.1. ${ }^{b}$ Values reported are for the final monoblock and diblock copolymers. 
Polymer 1 PS/DAP(12k) - NMR

12k-monoblk-ps/psDAP
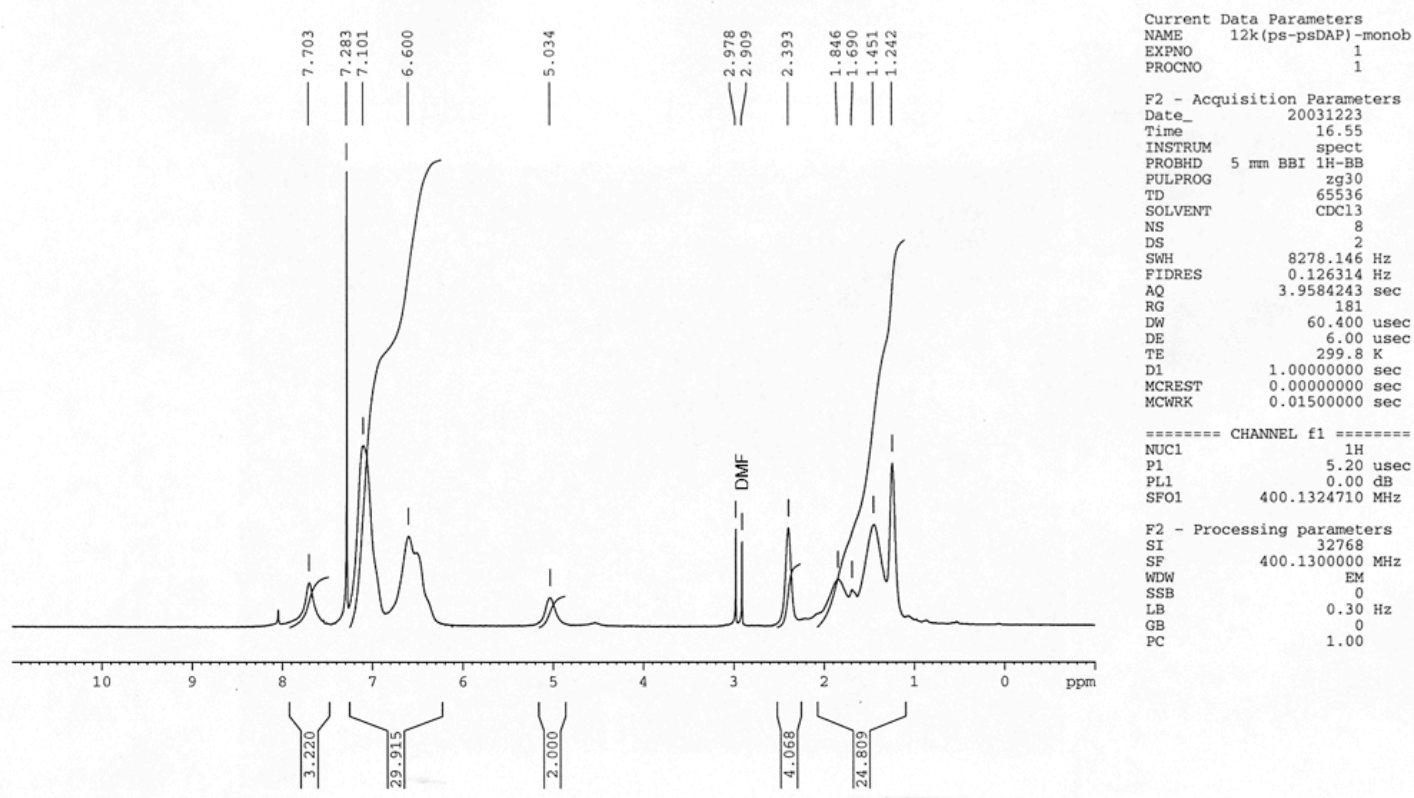

Polymer 1 chloromethylstyrene precursor PS/CMS(12k) - NMR

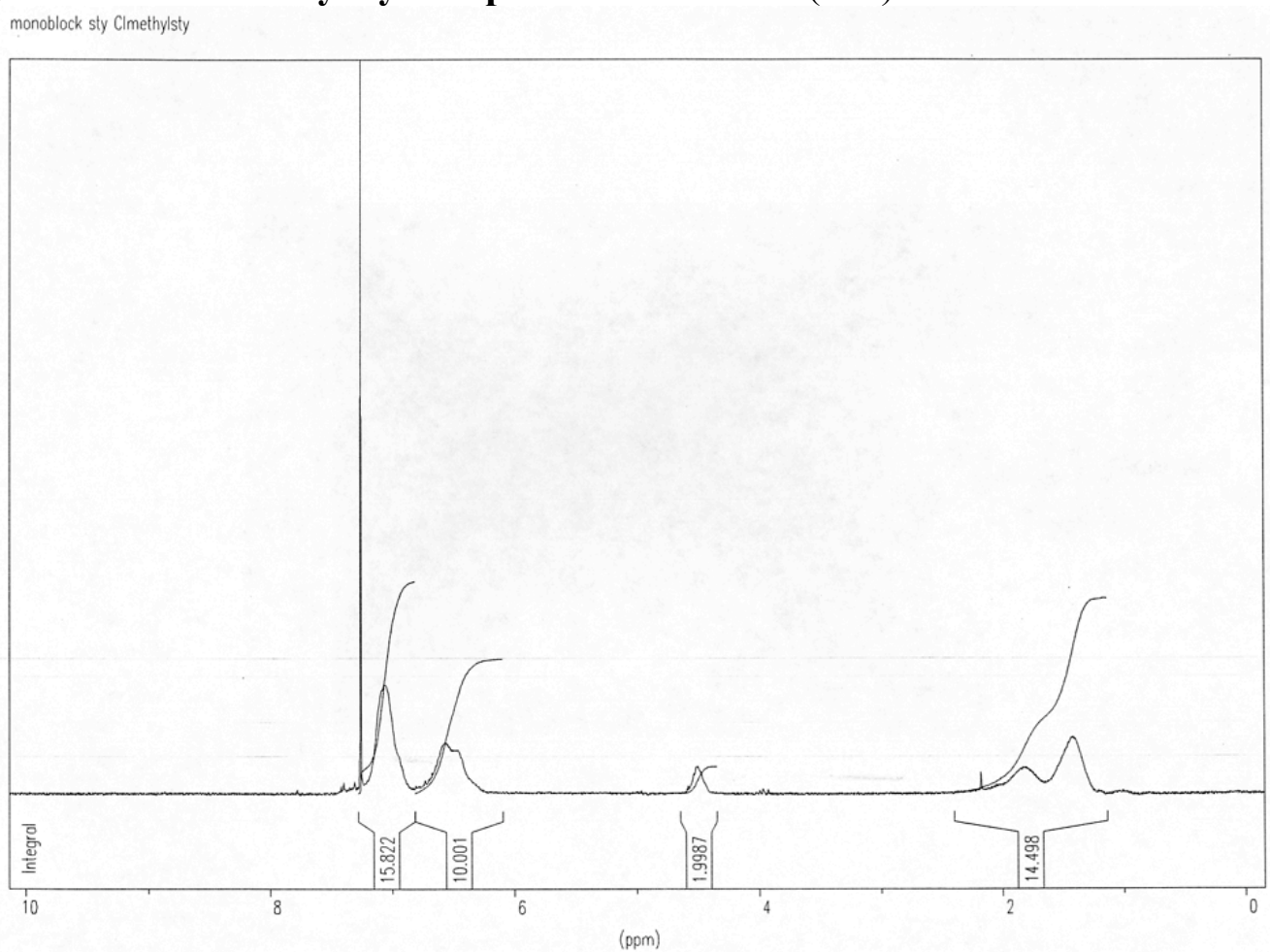


Polymer 1 chloromethylstyrene precursor PS/CMS(12K) - GPC

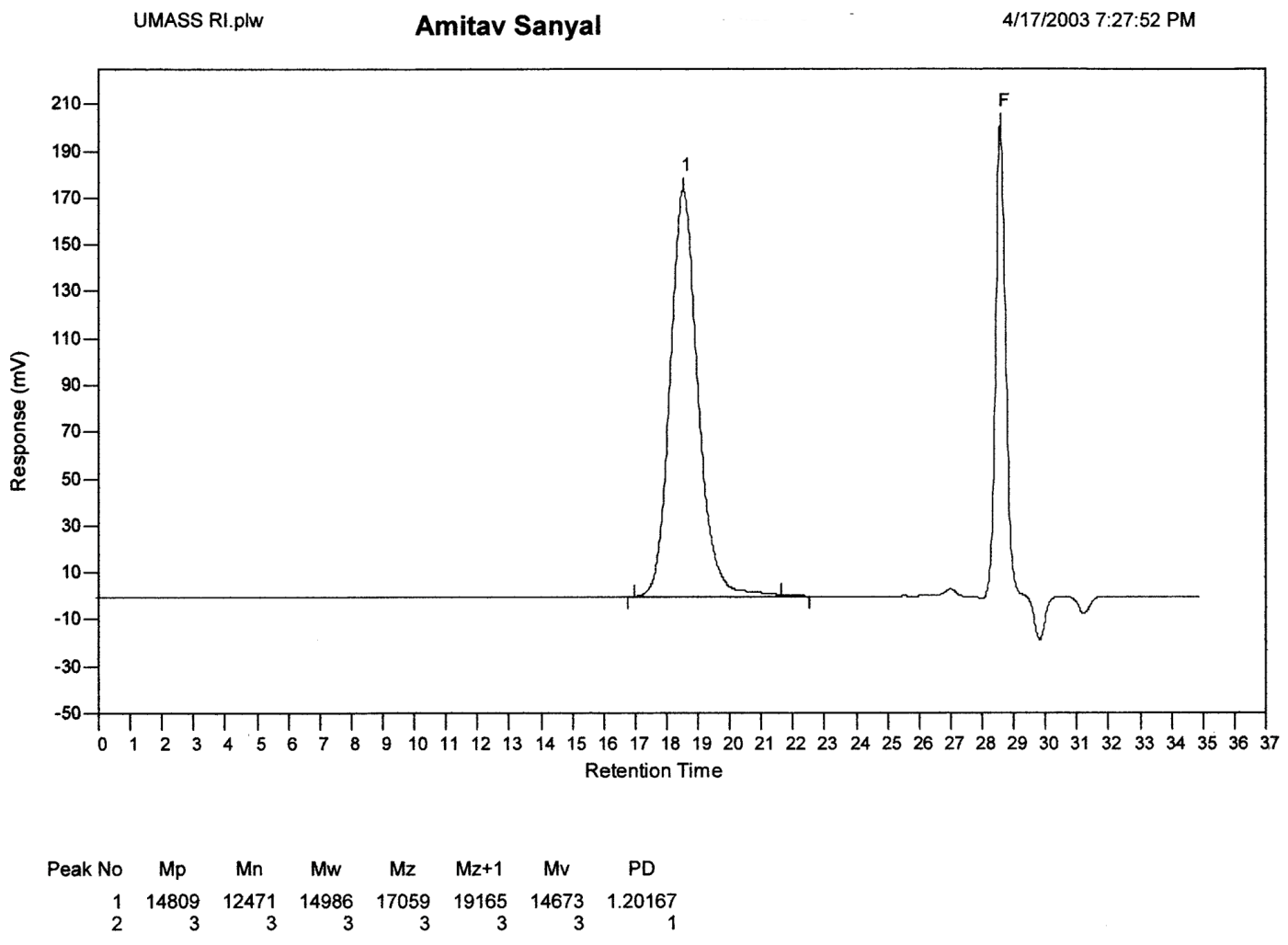

Polymer 2a PS/DAP(27k:7k) - NMR

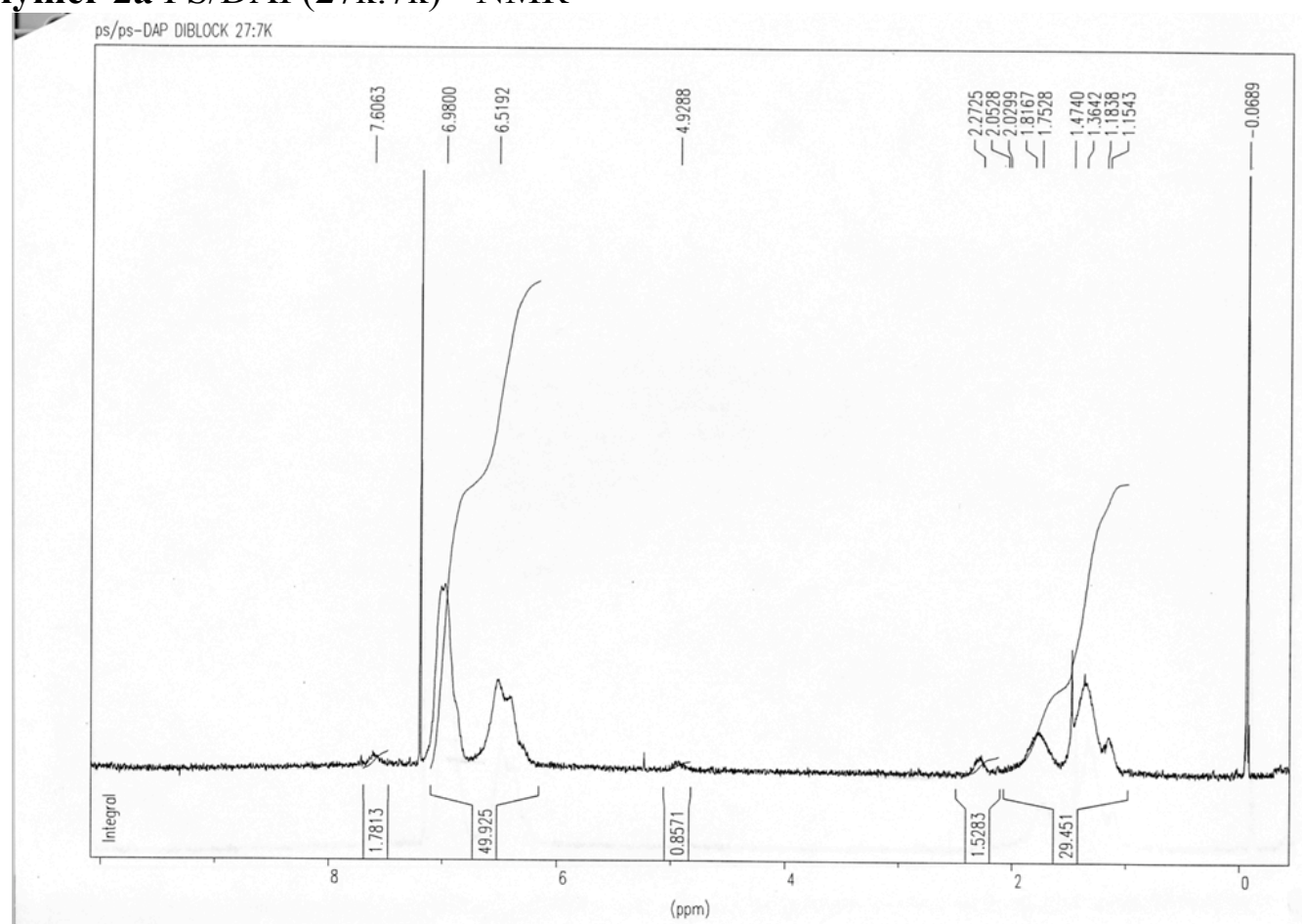


Polymer 2a chloromethylstyrene precursor PS/CMS(27k:7k) - NMR

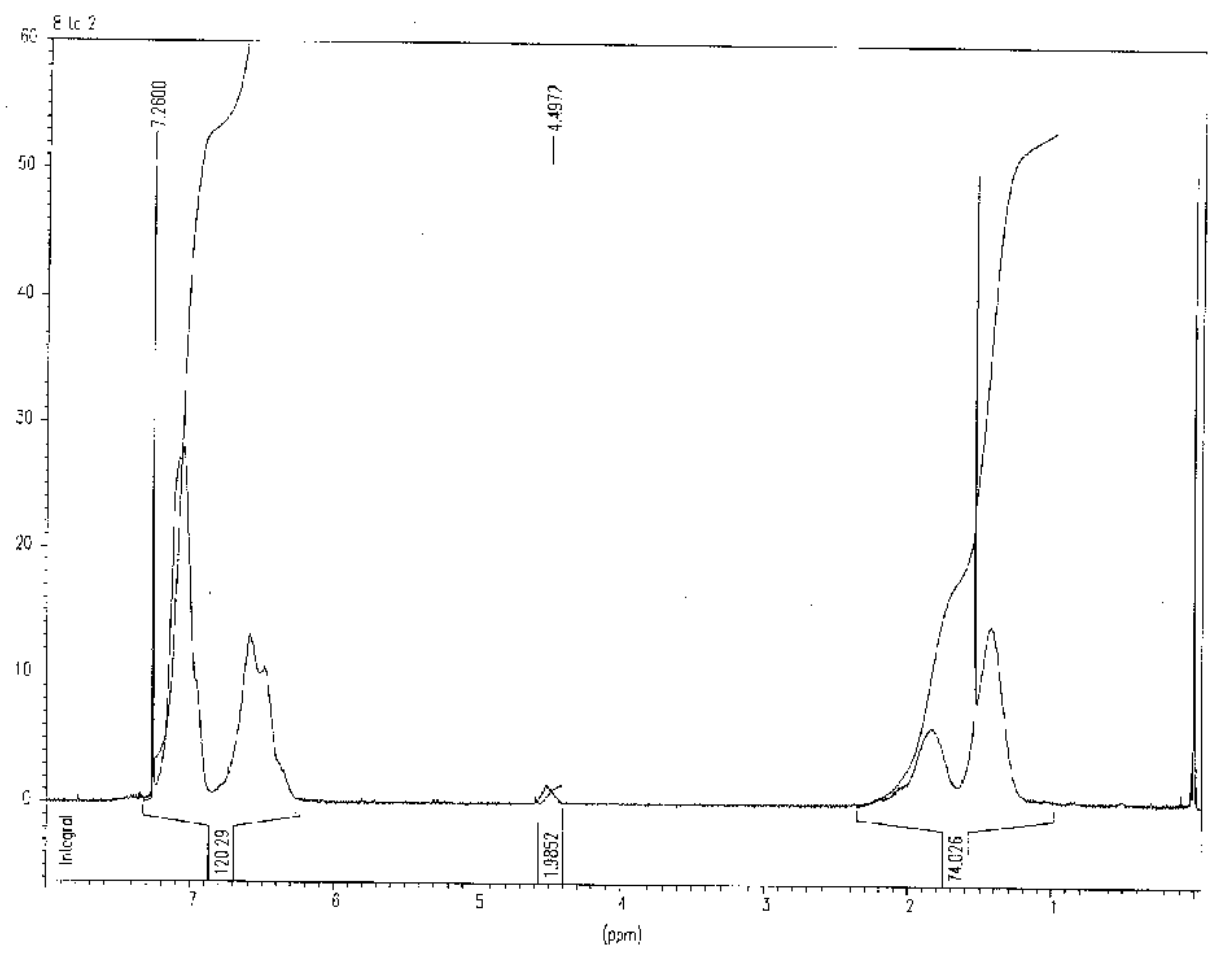

Polymer 2a chloromethylstyrene precursor PS/CMS(27k:7k) - GPC

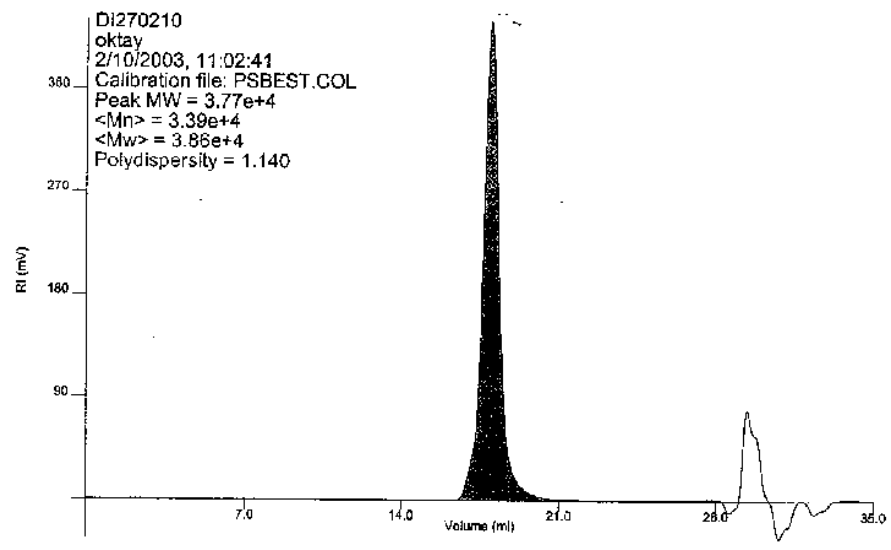




\section{Polymer 2b PS-DAP(27k:13k)-NMR}

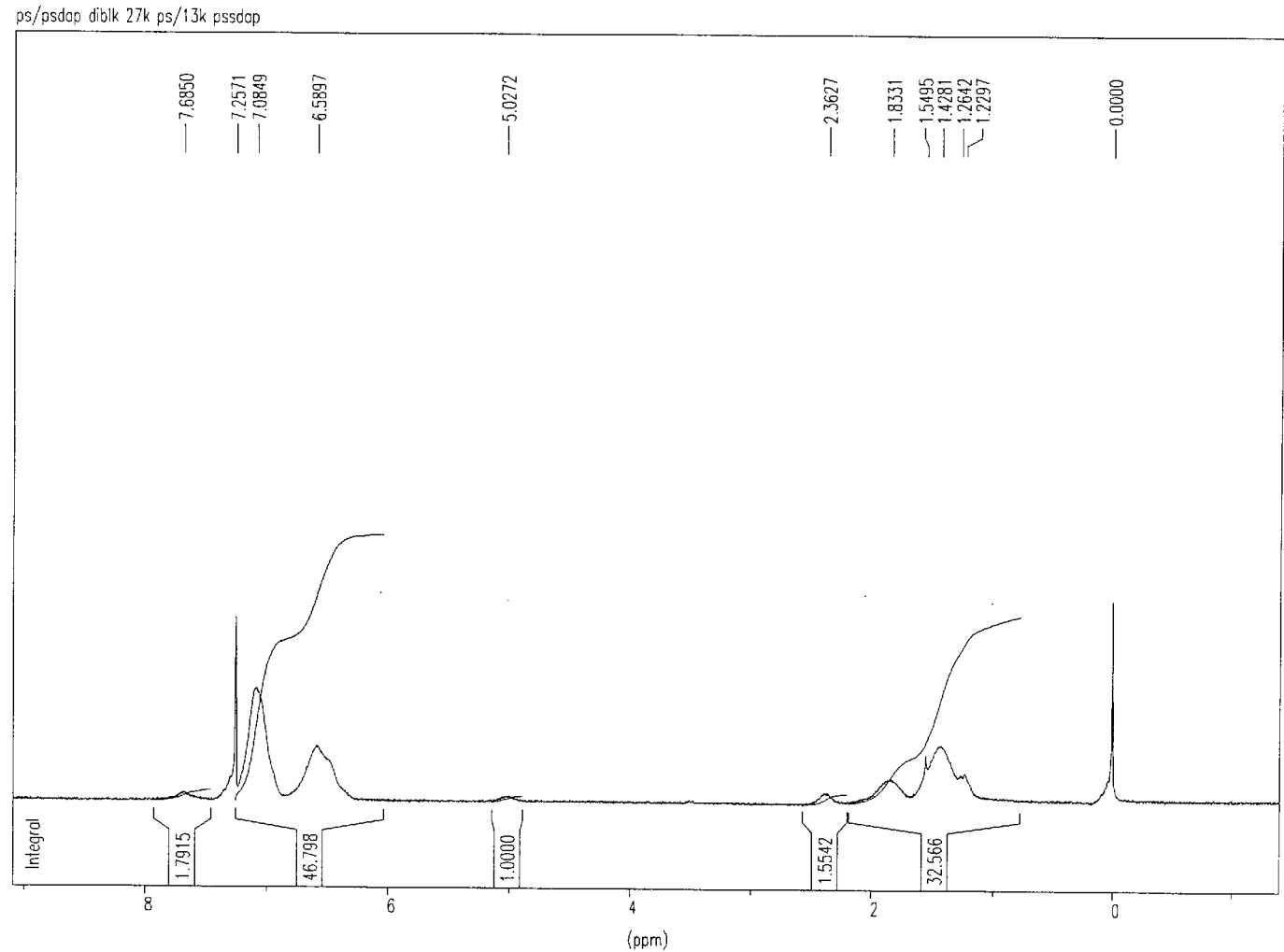

Polymer 2b chloromethylstyrene precursor PS-CMS(27k:13k)-NMR

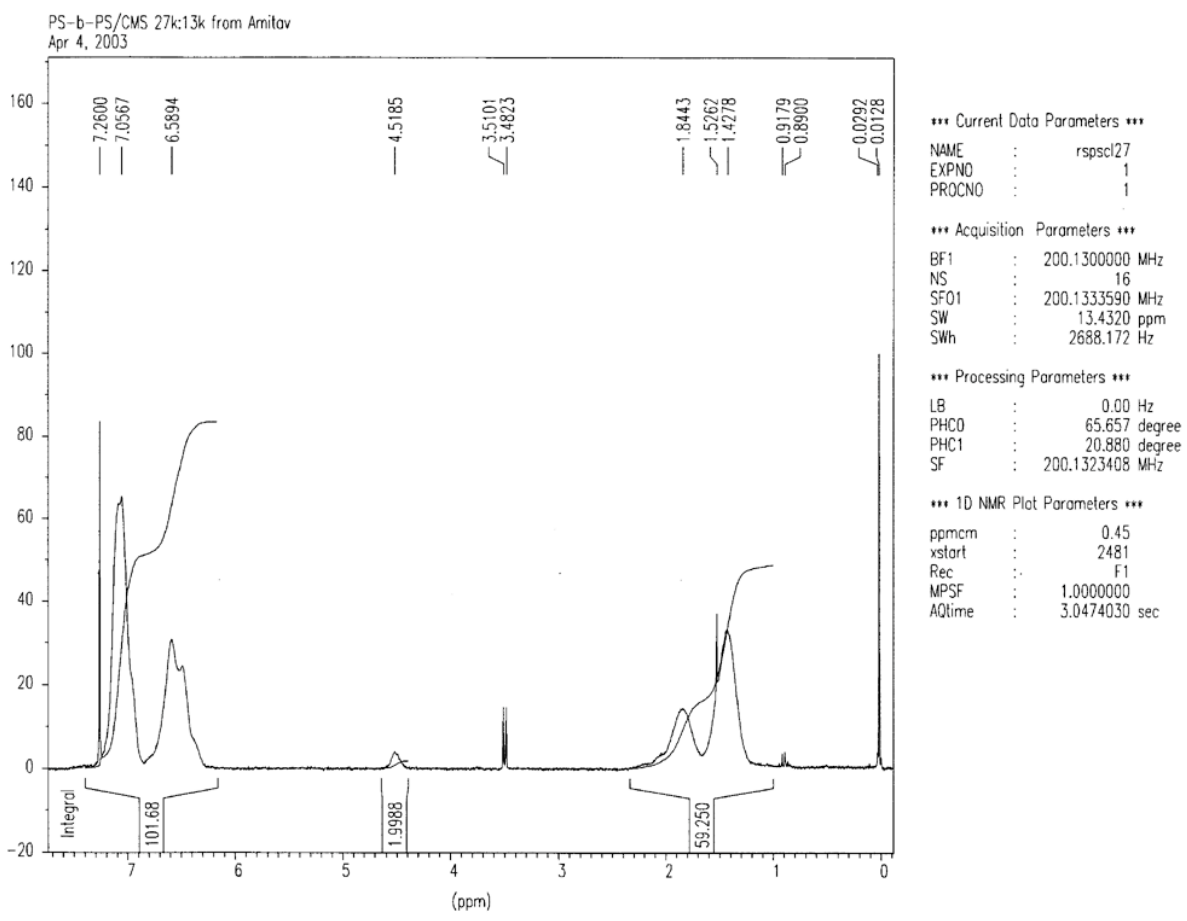


Polymer 2b chloromethylstyrene precursor PS-CMS(27k:13k)-GPC

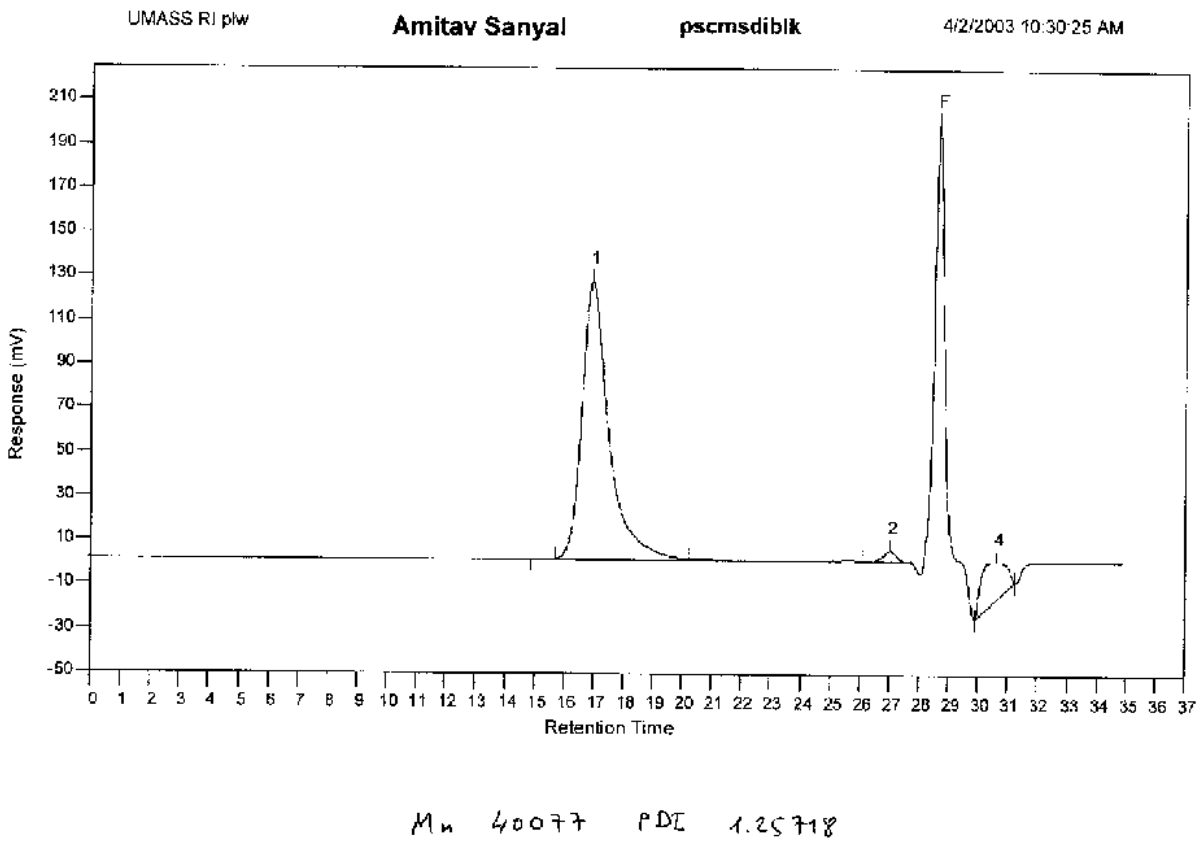

Polymer 2c PS/DAP(27k:27k) - NMR

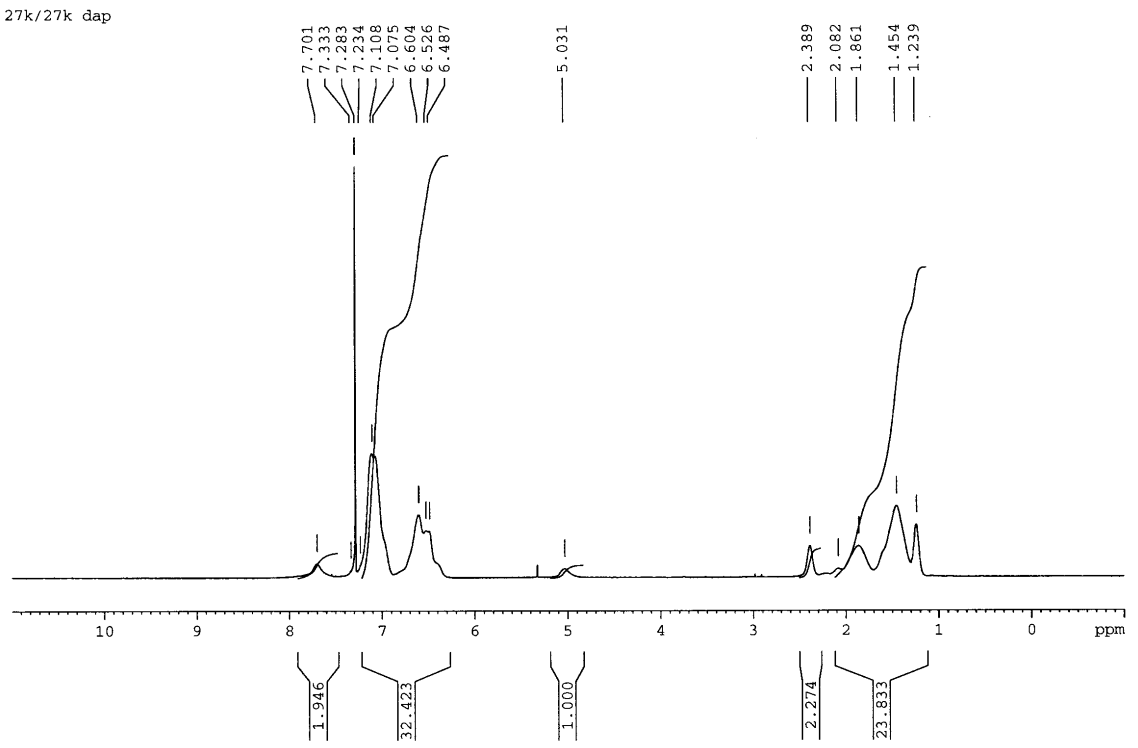

Current Data Parameters

$\begin{array}{ll}\text { F2 - } & \text { Acquisition Parameters } \\ \text { Date- } & 20040105 \\ \text { Time } & 14.02\end{array}$

INSTRUM
PROBHD $5 \mathrm{~mm}$ BBI $1 \mathrm{H}-\mathrm{BB}$

DS

FIDR

AQ

$\mathrm{DE}$
$\mathrm{TE}$
$\mathrm{D} 1$

MCRE

$===== \pm=$ CHANNEL f1 $1===== \pm=$

$\begin{array}{lr}\text { NUC1 } & 1 \mathrm{H} \\ \text { P1 } & 5.20 \mathrm{usec} \\ \text { PL1 } & 0.00 \mathrm{~dB} \\ \text { SE01 } & 400.1324710 \mathrm{MHz}\end{array}$

F2 - Processing parameters

$\begin{array}{ll}\mathrm{SI} & 32768 \\ \mathrm{SF} & 400.1300000 \mathrm{MHz}\end{array}$

SF
WDW
SSB
LB
GB
PC

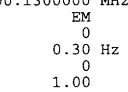


Polymer 2c chloromethylstyrene precursor PS/CMS(27k:27k)-NMR

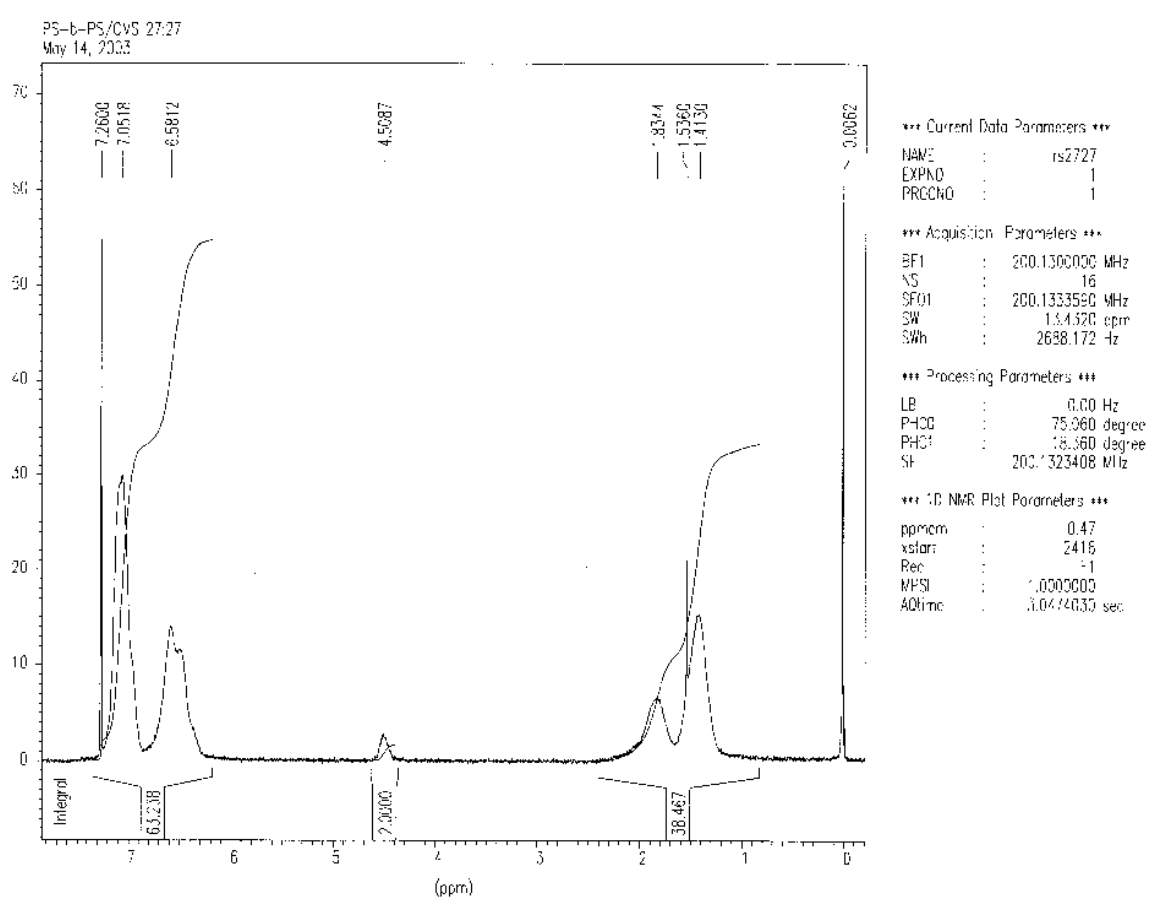

Polymer 2c chloromethylstyrene precursor PS-CMS(27k:27k)-GPC

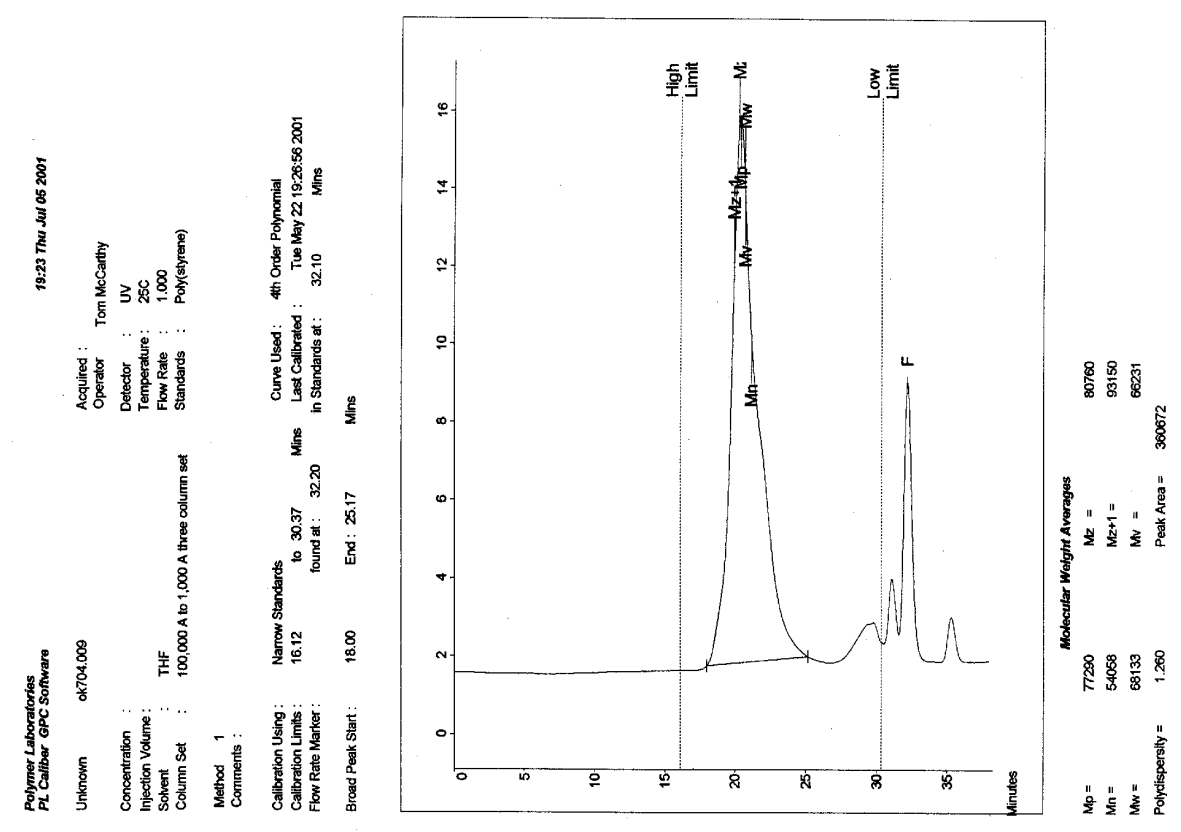


PS (27k)-GPC

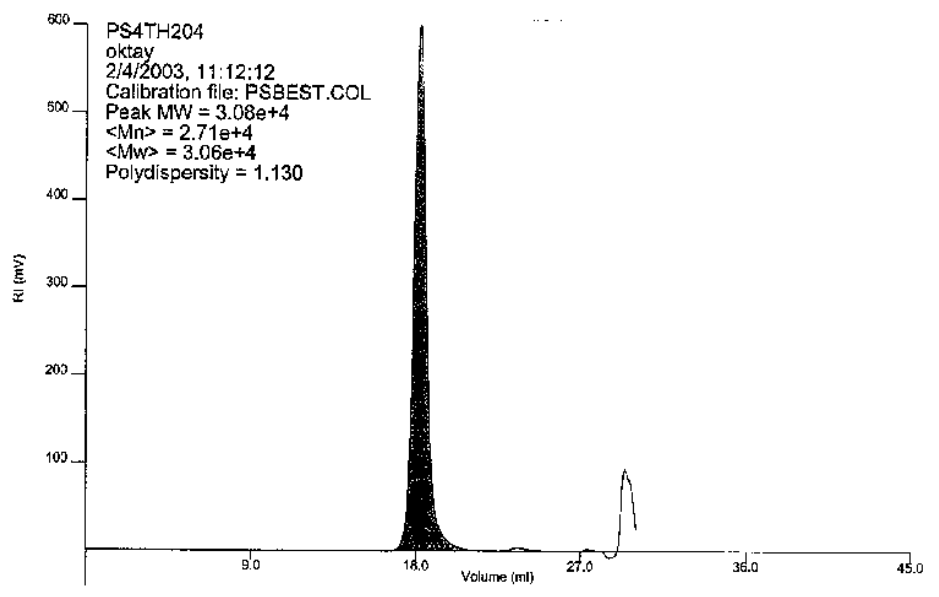

Polymer 2d PS/DAP(13k:13k) - NMR
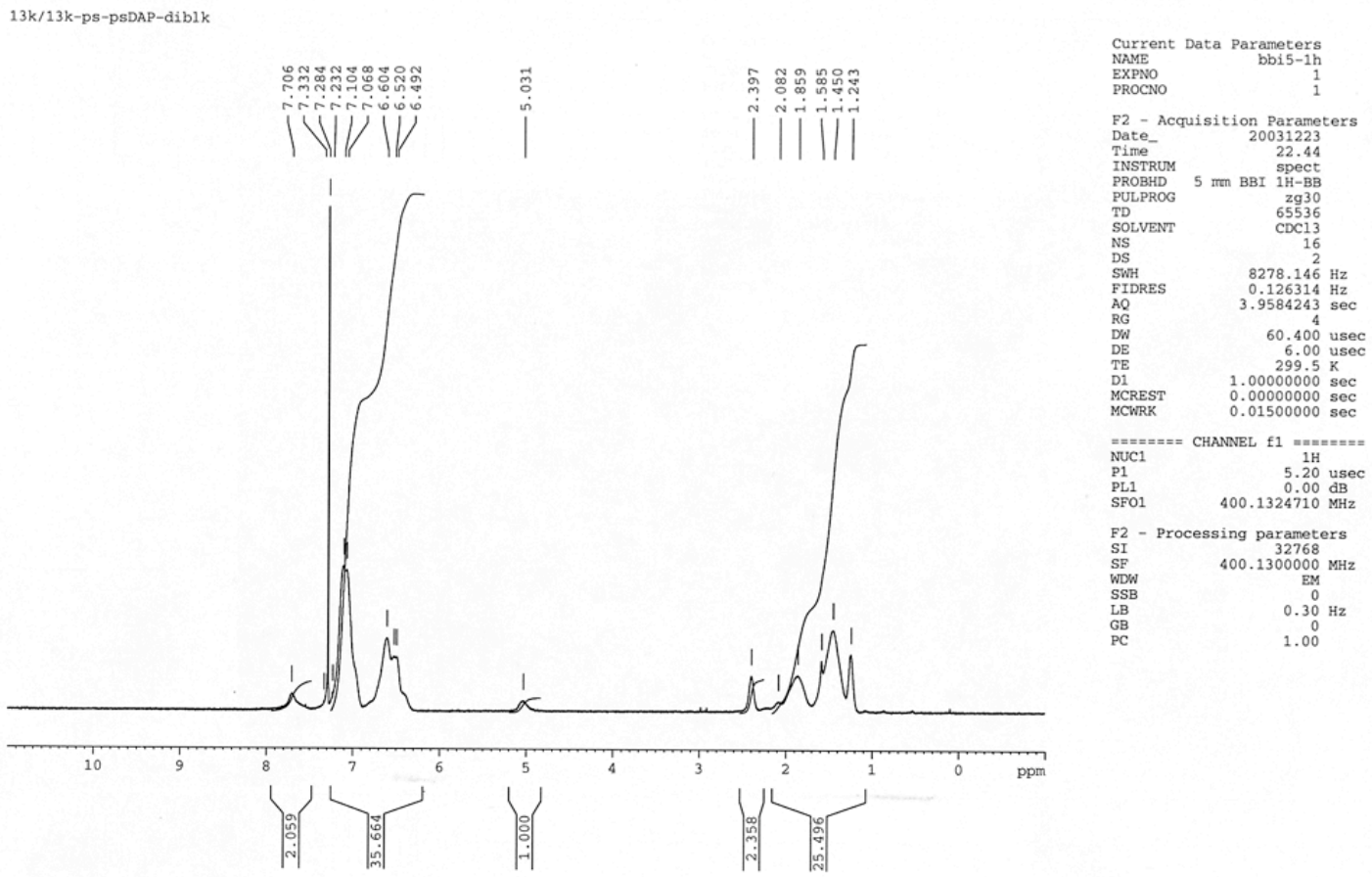
Polymer 2d chloromethylstyrene precursor PS/CMS(13k:13k) - NMR

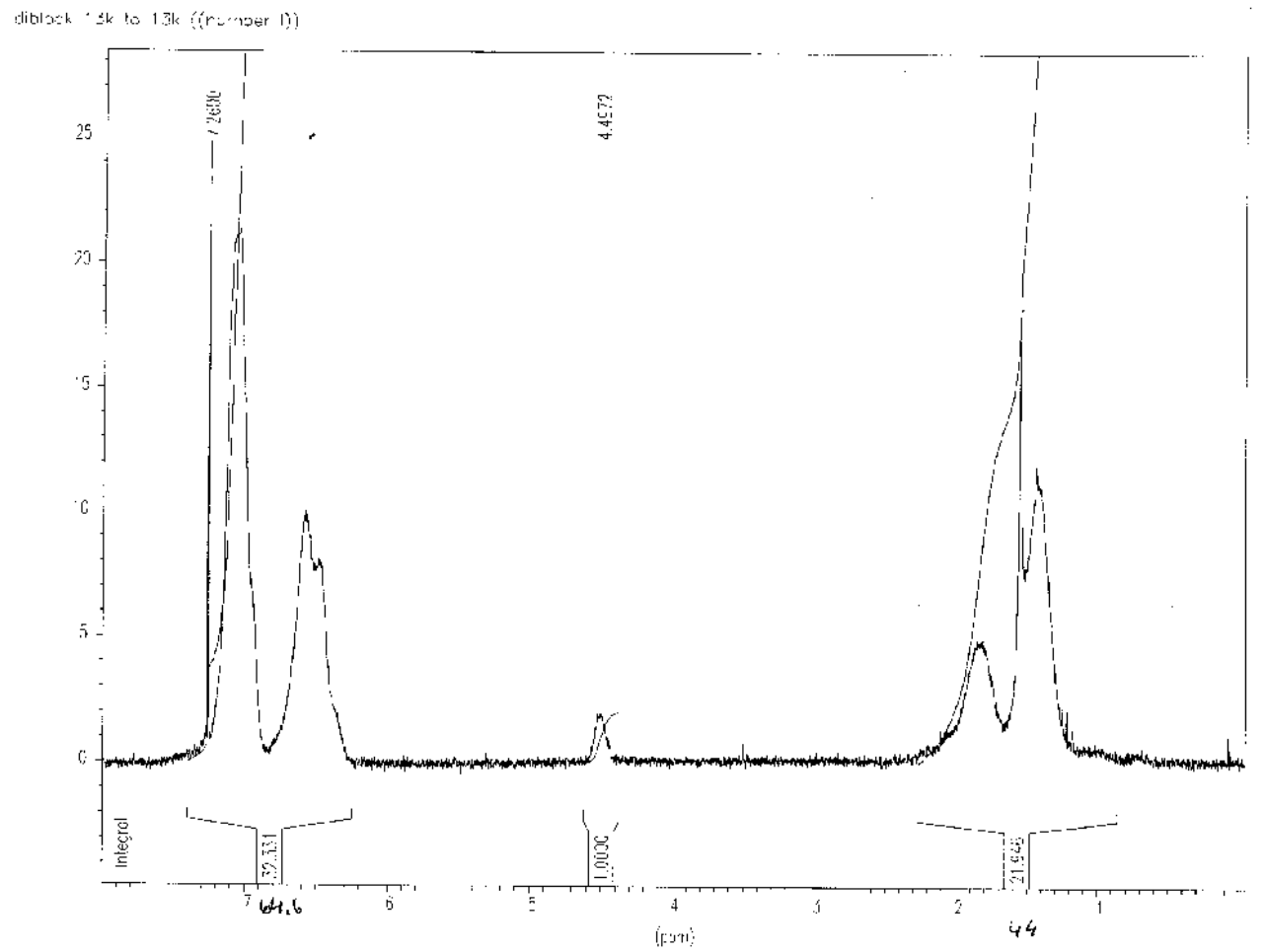

Polymer 2d chloromethylstyrene precursor PS/CMS(13k:13k) - GPC

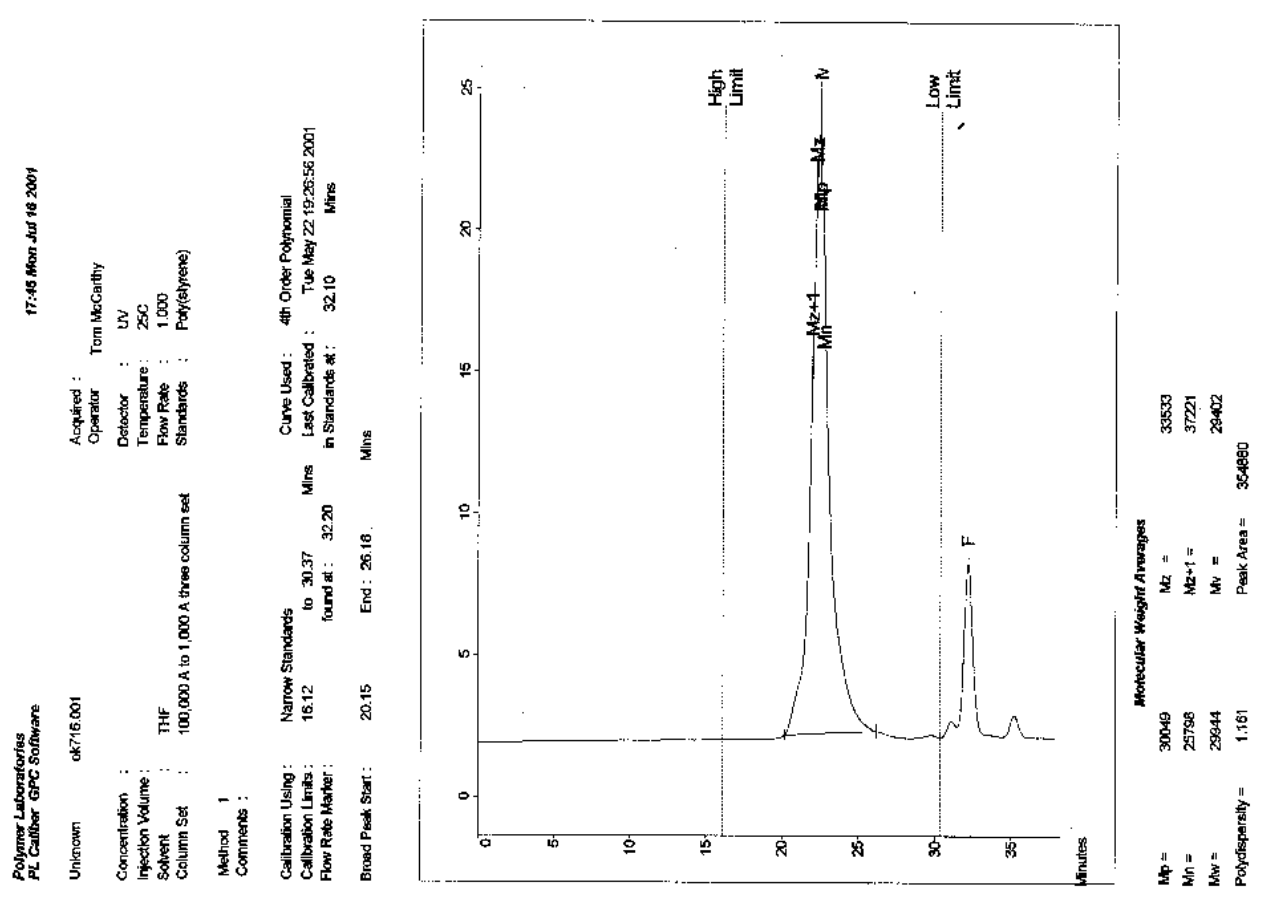


Polymer 4 Poly(4-F-Styrene)/(PS-DAP) (29K/11K) - NMR

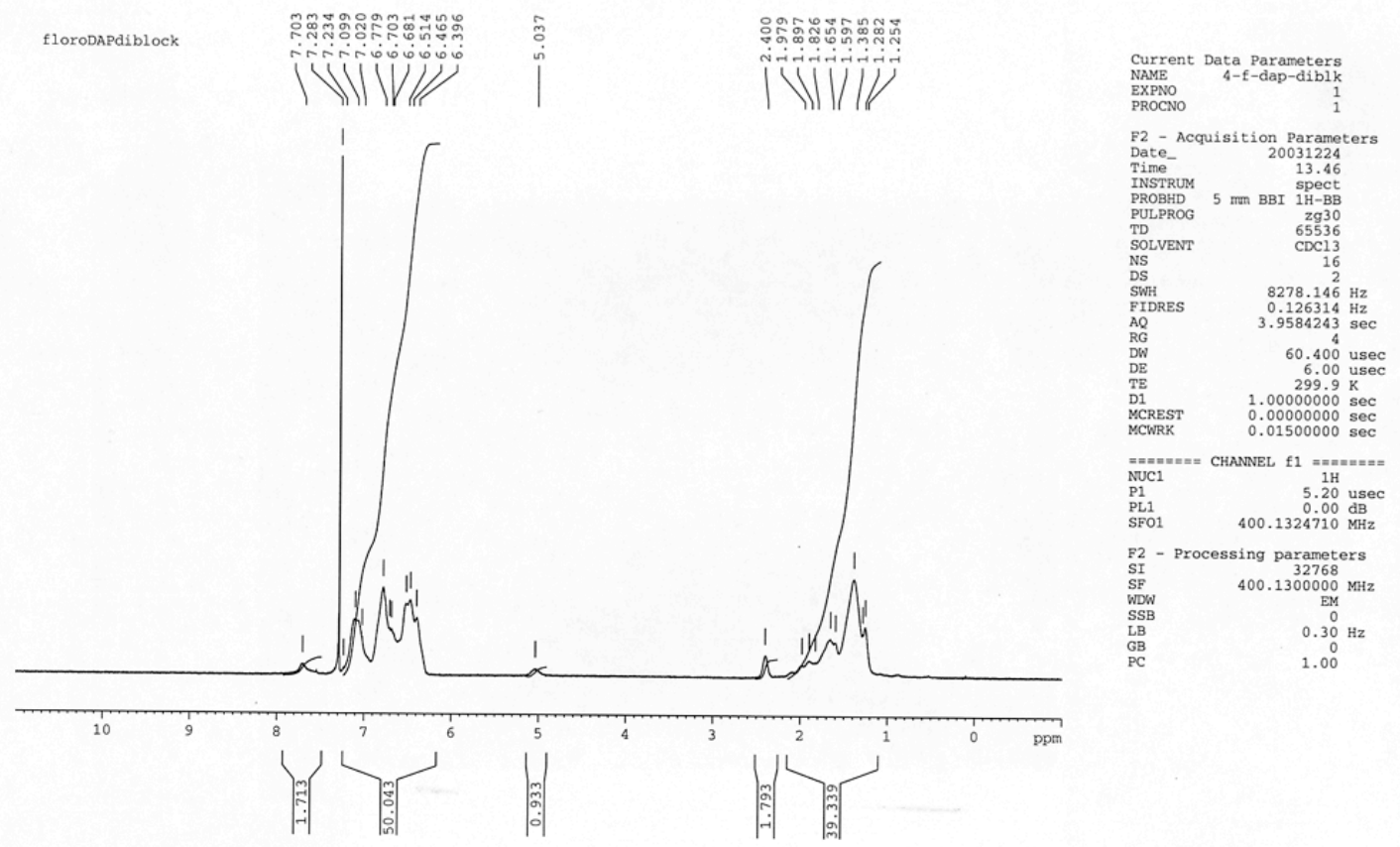

Polymer 4 chloromethylstyrene precursor Poly(4-F-Styrene)/(PS-CMS)(29K/11K) NMR
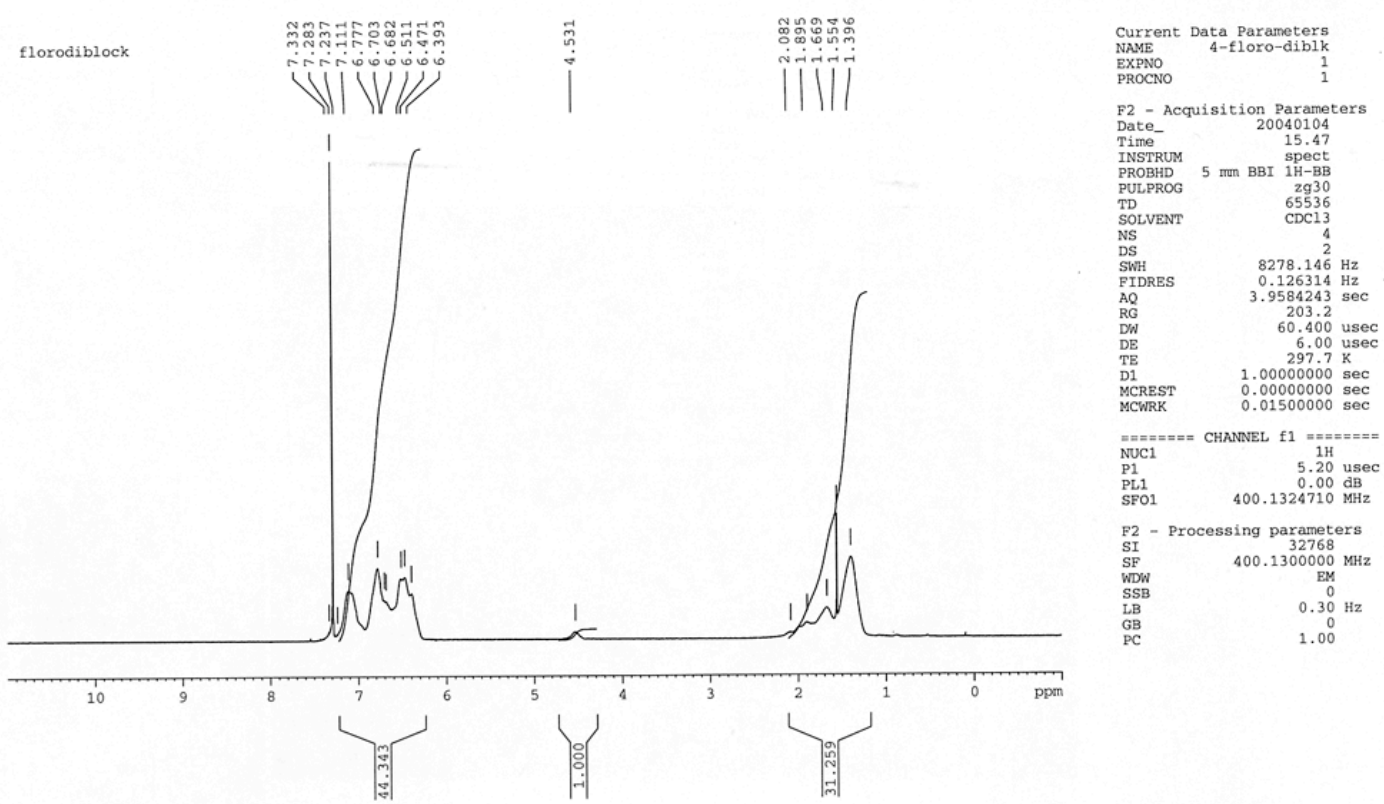
Polymer 4 chloromethylstyrene precursor Poly(4-F-Styrene)/(PS-CMS) (29K/11K) GPC

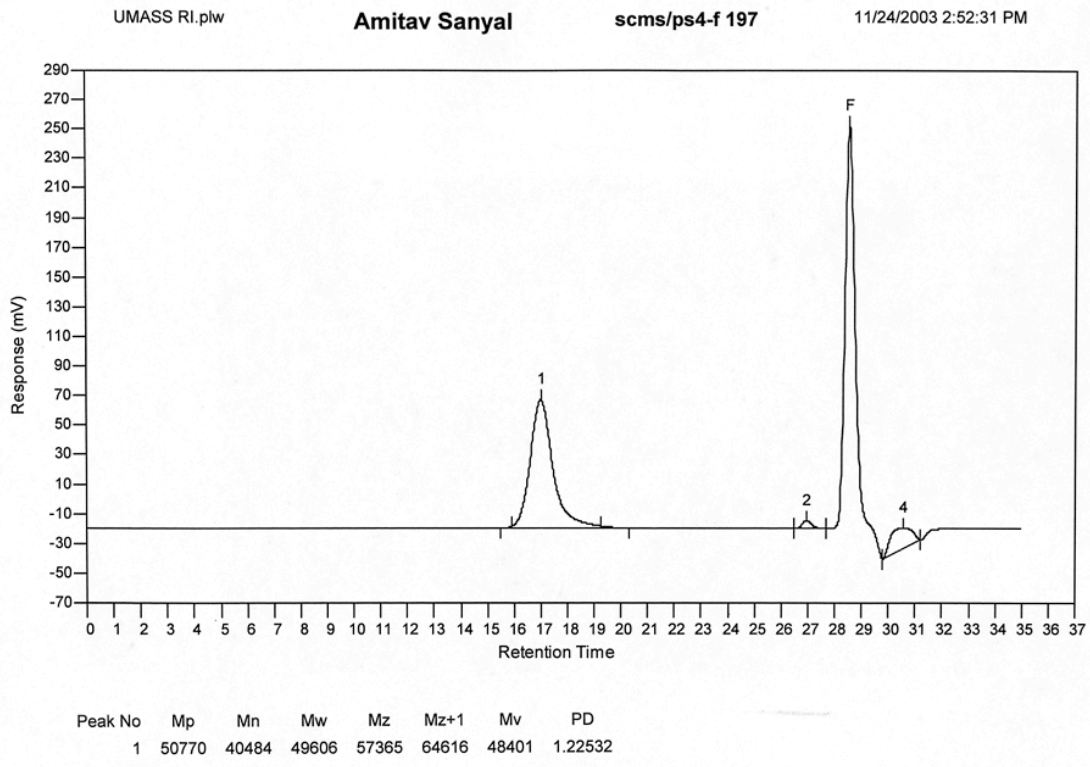

Polymer 4 (Block A) Poly(4-F-Styrene) - NMR
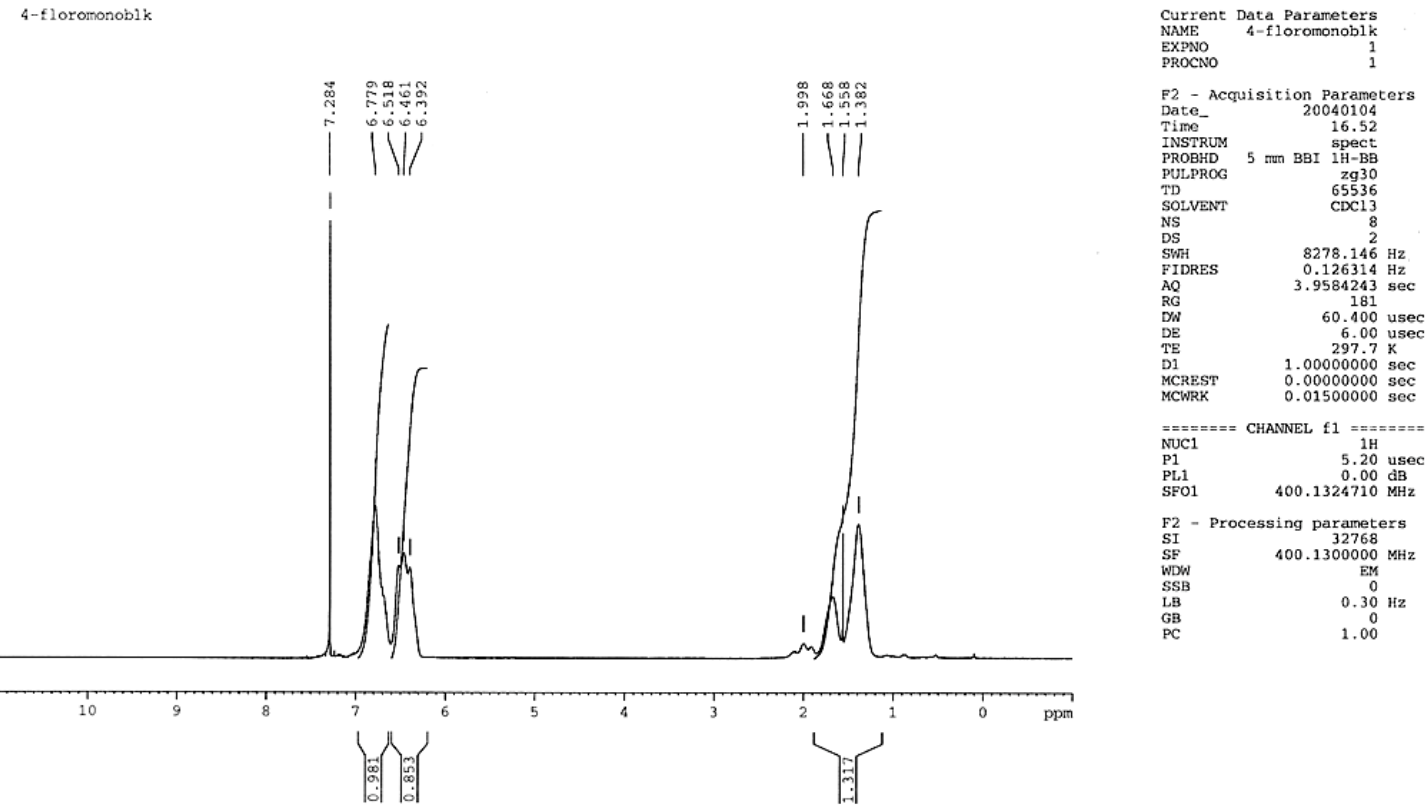
Polymer 4 (Block A) PS(4-F-Styrene) - GPC

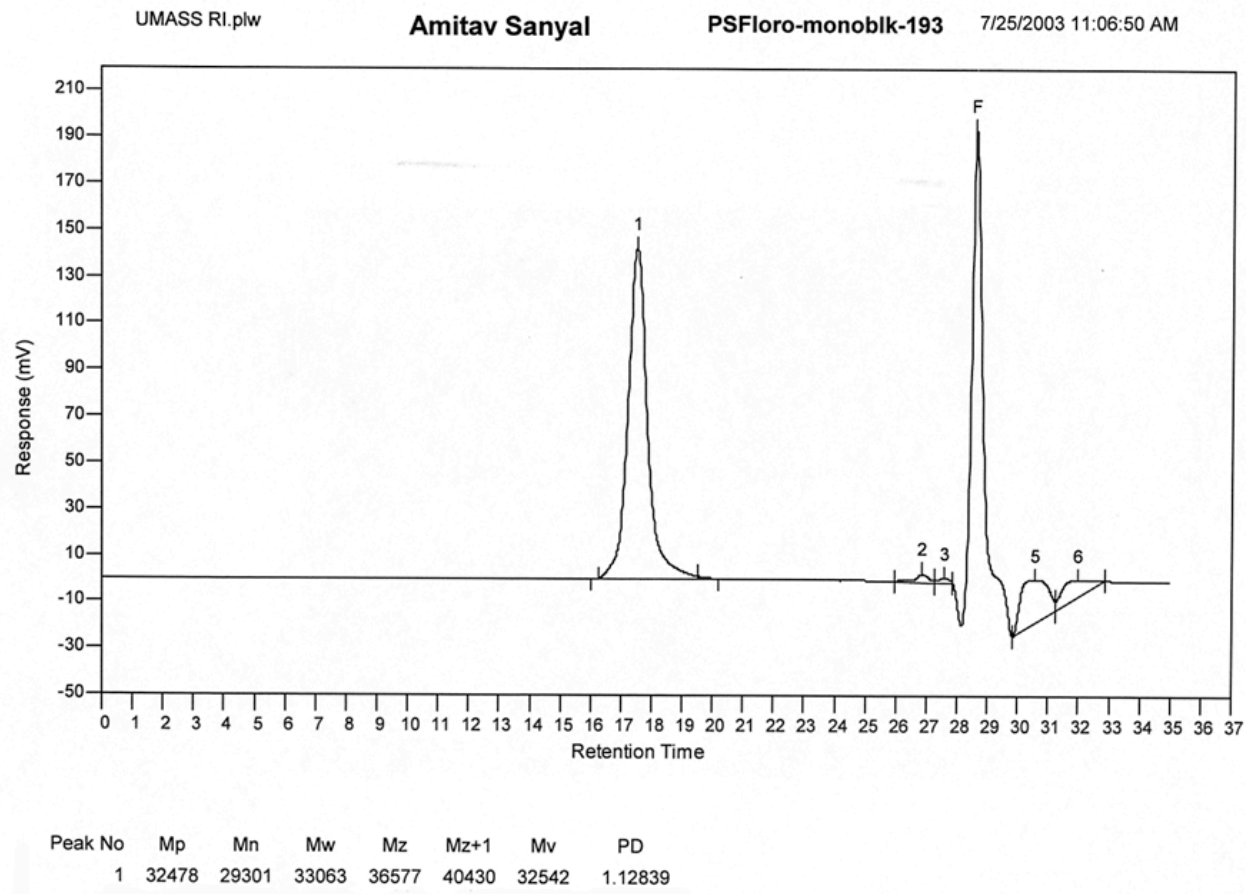

